\title{
Calibración de un modelo lluvia-escorrentía combinando la optimización manual y automática para un sistema de alta montaña
}

\author{
Calibration of a conceptual rainfall-runoff model combining manual and automatic \\ optimization for high mountain systems
}

Ismael Orozco ${ }^{1 *}$, Aldo I. Ramírez ${ }^{2}$, Félix Francés ${ }^{3}$ 1 Departamento de Ingeniería Geomática e Hidráulica, Universidad de Guanajuato. Av. Juárez No. 77, Zona Centro, C.P. 36000,

Guanajuato, Gto., México. Correo electrónico: i.orozco@ugto.mx ${ }^{2}$ Escuela de Ingeniería y Ciencias, Tecnológico de Monterrey. 3 Instituto de Ingeniería del Agua y Medio Ambiente, Universitat Politècnica de València. *Autor de correspondencia

\section{Resumen}

\begin{abstract}
La calibración de un modelo lluvia-escorrentía es un proceso complicado que puede llevar mucho tiempo y esfuerzo. Su éxito está condicionado a la experiencia del modelador y al conocimiento que se tenga del sistema físico que se desea modelar. Por ello, en este trabajo, se propone una metodología que combina la calibración manual y automática para obtener los parámetros efectivos de un modelo hidrológico aplicado a una cuenca de alta montaña. Lo anterior se debe a que calibrar simultáneamente los parámetros hidrológicos y los parámetros de fusión de nieve puede no representar adecuadamente las condiciones reales de los sistemas de alta montaña. La metodología propuesta ha sido probada usando el modelo hidrológico distribuido TETIS desarrollado en la Universitat Politècnica de València. Los resultados obtenidos son buenos al alcanzarse índices de eficiencia de Nash-Sutcliffe cercanos a 0.9 en calibración y a 0.8 en validación, con parámetros dentro de los rangos establecidos en la literatura científica.
\end{abstract}

Palabras clave: Calibración; algoritmo de optimización; modelación hidrológica; fusión de nieve; método grado-día.

\begin{abstract}
The calibration of a conceptual rainfall-runoff model is a complicated process that can take a long time and effort. Its success depends on the experience of the modeler and on the knowledge of the basin. For this reason, in this paper, a combination of manual and automatic calibration has been proposed to obtain the effective parameters of a hydrological model applied to a high mountain basin. This is because calibrating hydrological parameters and snow melting parameters simultaneously may not adequately represent the real conditions of high mountain systems. The methodology proposed in this paper has been implemented using the distributed hydrological TETIS model developed at the Universitat Politècnica de València. The results obtained are acceptable, because the Nash-Sutcliffe efficiency index is reached close to 0.9 in calibration and 0.8 validation, with parameters within the ranges established in the scientific literature.
\end{abstract}

Keywords: Calibration; optimization Algorithm; hydrological modeling; snowmelt; degree-day method.

Como citar: Orozco, I., Ramírez, A. I., \& Francés, F. (2019). Calibración de un modelo lluvia-escorrentía combinando la optimización manual y automática para un sistema de alta montaña. Acta Universitaria 29, e2187. doi. http://doi.org/10.15174.au.2019.2187 


\section{Introducción}

En términos generales, el objetivo de la calibración de un modelo puede ser definido como la selección de los parámetros que permitan simular el comportamiento hidrológico de una cuenca de la mejor manera posible (Madsen, 2000; Yapo, Gupta \& Sorooshian, 1998). Para ello, existe la calibración manual y automática, basada esta última en algoritmos de optimización (Chapman, 1970; Dawdy \& O'Donnell, 1965; Gupta \& Sorooshian, 1985; Hendrickson, Sorooshian \& Brazil, 1988; Johnston \& Pilgrim, 1976; Monro, 1971; Nash \& Sutcliff, 1970; Pickup, 1977; Sorooshian, 1981; Sorooshian, Duan \& Gupta, 1983). En el caso de la calibración manual, Eckhardt \& Arnold (2001) comentan que su éxito depende esencialmente de la experiencia del modelador, de su conocimiento e interacción con el modelo y el sistema que se desea modelar. La calibración manual es subjetiva y puede consumir mucho tiempo. Por otro lado, la calibración automática puede estar sujeta a los principales problemas que afectan a los algoritmos automáticos y que, según Duan, Sorooshian \& Gupta (1992), son la existencia de múltiples regiones de atracción, donde el óptimo encontrado depende del valor inicial; la obtención de eficiencias similares con diferentes parámetros; y la dependencia de la función objetivo seleccionada. Por lo anterior, la calibración de un modelo lluvia-escorrentía es un proceso complicado en el que se cree que deben interactuar ambas formas de calibración, y más tratándose de una cuenca de alta montaña, en la cual interactúan los principales componentes de producción de la escorrentía y la variable nieve que influye significativamente en el almacenamiento temporal y la liberación del agua (Jansson, Hock \& Schneider, 2003). Según Hock (2003), el éxito de la modelación de escorrentía en cuencas de alta montaña depende, en gran medida, de la cuantificación que se hace del proceso de fusión de nieve. Por tanto, la simulación de estos sistemas resulta más compleja en los casos donde la variable nieve puede tomar un papel protagónico en las crecidas, al combinarse un incremento rápido de las temperaturas y lluvias fuertes. En países como USA y Canadá, la mayor parte de Europa y el norte de Asia, la producción de escorrentía proveniente de la fusión de nieve que puede alcanzar hasta un 90\% del caudal anual (Gray, 1970). De lo anterior se desprende el gran interés por modelar estos sistemas a través de modelos determinísticos como una alternativa de gestión y control de inundaciones.

\section{Área de estudio}

El área de estudio seleccionada es la subcuenta del afluente norte de la cuenca del río American que se localiza en Sierra Nevada USA, entre los estados de California y Nevada (Longitud $118^{\circ}-124^{\circ} \mathrm{W}$ y latitud $38^{\circ}$ $40^{\circ} \mathrm{N}$ ). La subcuenca tiene un área de $886 \mathrm{~km}^{2}$ (figura 1). 


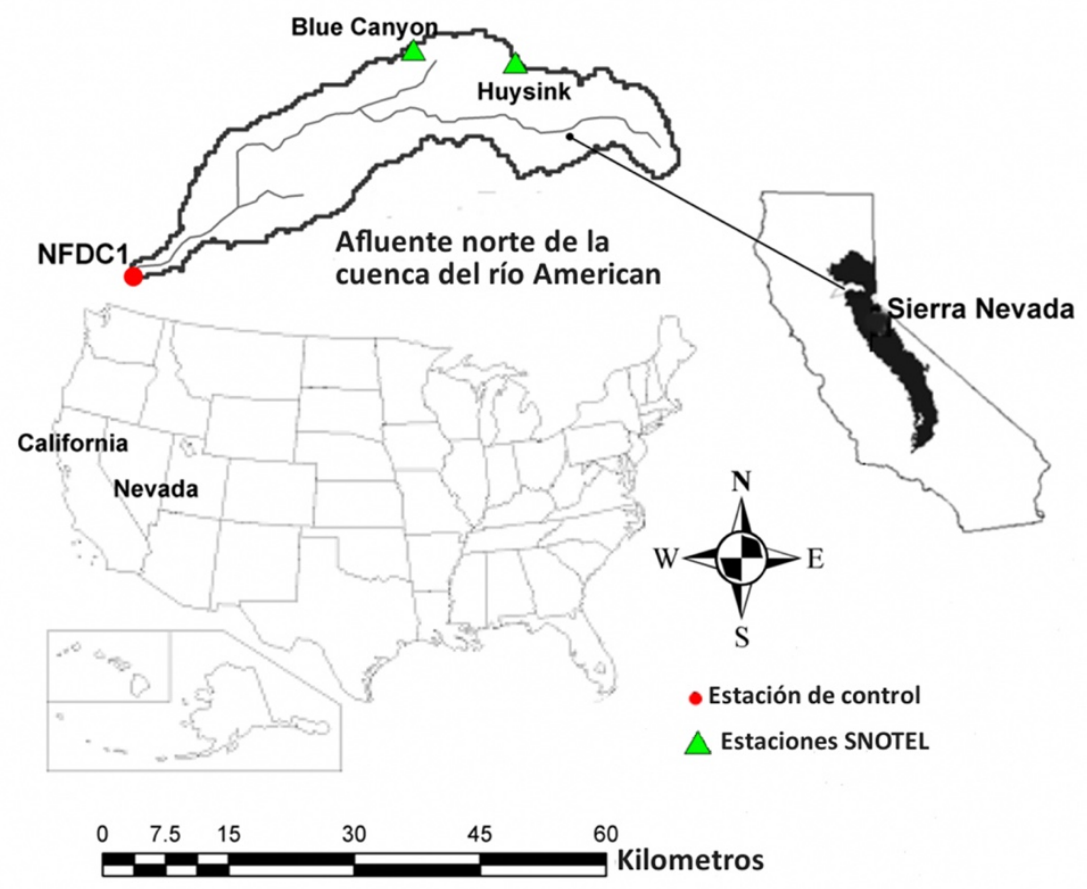
hidrométrica NFDC1 y $\boldsymbol{\Delta}$ estaciones de nieve SNOTEL).

Fuente: Elaboración propia.

La subcuenca se ubica entre los 281 m.s.n.m. y los 2630 m.s.n.m., por lo cual su precipitación se produce en forma de lluvia, nieve y la combinación de ambas (Jeton, Dettinger \& Smith, 1996; Shamir \& Georgakakos, 2007). La precipitación acumulada media anual varía de $813 \mathrm{~mm}$ a $1651 \mathrm{~mm}$. El periodo de invierno comprende seis meses de noviembre a abril, registrándose temperaturas promedio entre los $0{ }^{\circ} \mathrm{C}$ $18^{\circ} \mathrm{C}$ (Pandey, Cayan \& Georgakakos, 1999).

\section{Modelo de lluvia-escurrimiento}

En esta investigación se ha utilizado el modelo lluvia-escorrentía TETIS desarrollado en el Instituto de Ingeniería del Agua y Medio Ambiente (IIAMA), de la Universitat Politècnica de València. Es un modelo con parámetros físicamente basados que simula de manera distribuida los principales procesos del ciclo hidrológico realizando un balance hídrico a nivel de celda (Francés, Vélez \& Vélez, 2007). Es un modelo que puede descargarse del siguiente link: http://lluvia.dihma.upv.es/ES/software/currentreleases.html. El modelo utiliza un esquema conceptual a nivel de celda que incluye seis tanques, seis variables de estado inicial, cinco flujos de salida y cinco nodos de control conectados verticalmente (figura 2). 


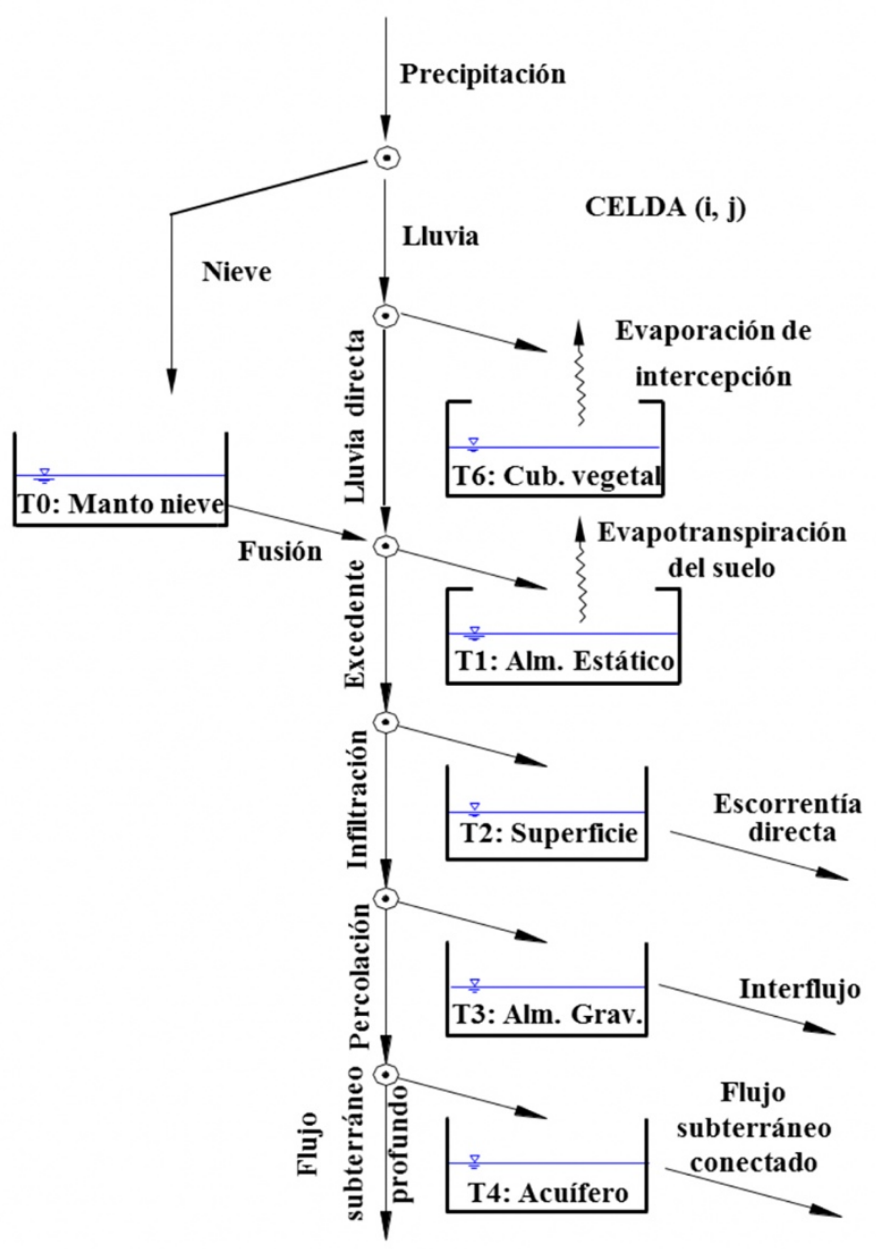

Figura 2. Esquema conceptual del movimiento vertical a nivel de celda del modelo TETIS. Fuente: Francés et al., 2014.

En la construcción del modelo se ha usado un Modelo Digital de Elevaciones (MDE) con una resolución espacial de 400 m (figura 3), obtenido del Distributed Hydrologic Model Intercomparison Project-Phase 2 (DMIP2) de la National Oceanic and Atmospheric Administration's National Weather Service (NOAA/NWS) [http://www.nws.noaa.gov/oh/hrl/dmip/2]. 


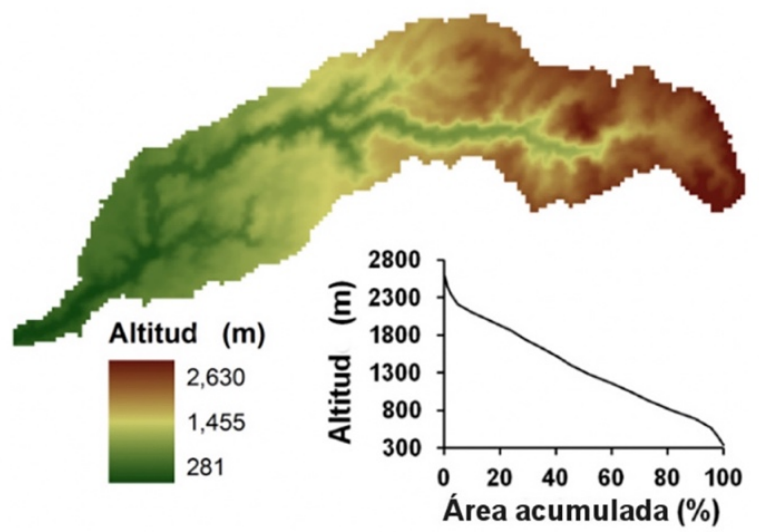

Figura 3. Modelo digital de elevaciones y curva hipsométrica de la subcuenca del río American. Fuente: Elaboración propia.

A partir del MDE, se ha realizado la caracterización de la hidromorfometría de la subcuenca del río American (figura 4).

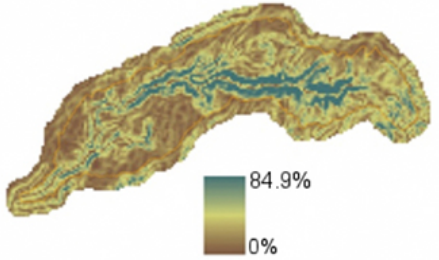

a)

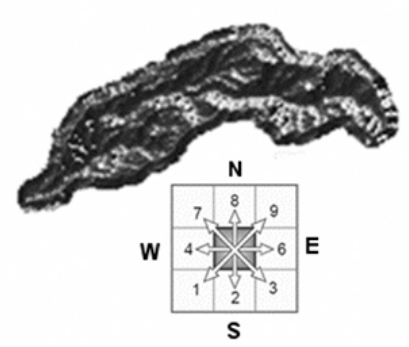

b)

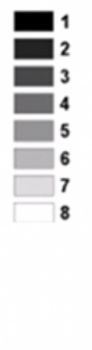

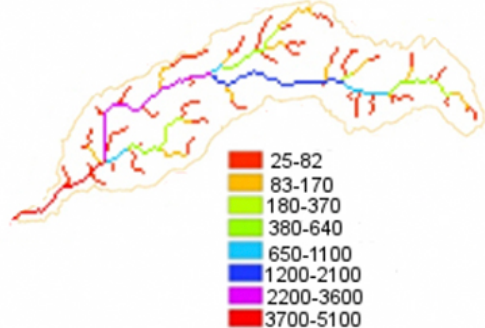

c)

Figura 4. a) Mapa de pendientes, b) mapa de direcciones de flujo y c) mapa de acumulación de flujo. Fuente: Elaboración propia.

Los parámetros de almacenamiento estático $\left(\mathrm{H}_{\mathrm{u}}\right)$, conductividad hidráulica del suelo $\left(\mathrm{k}_{\mathrm{s}}\right)$ y conductividad hidráulica en el substrato rocoso ( $\left.k_{p}\right)$ (figura 5) se han estimado empleando información ráster de edafología, geología y cubierta vegetal, con una resolución espacial de un kilómetro. La información utilizada para calcular los parámetros ha sido obtenida del DMIP2 (Smith et al., 2013). 

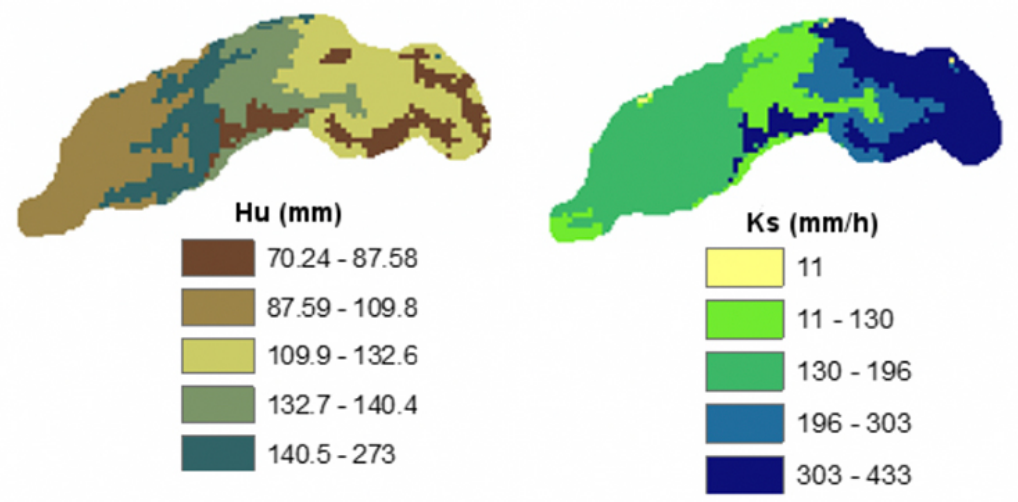

Los datos de entrada de precipitación y temperatura utilizados consisten en series horarias obtenidos también del DMIP2 en formato NEXRAD (Moreda, Cong, Schaake \& Smith, 2006), con una resolución espacial de $4762.5 \mathrm{~m}$ (figura 6). Para reducir los tiempos de cómputo en las simulaciones se recortó la malla principal a una malla de solo 13 renglones y 23 columnas (figura 6).

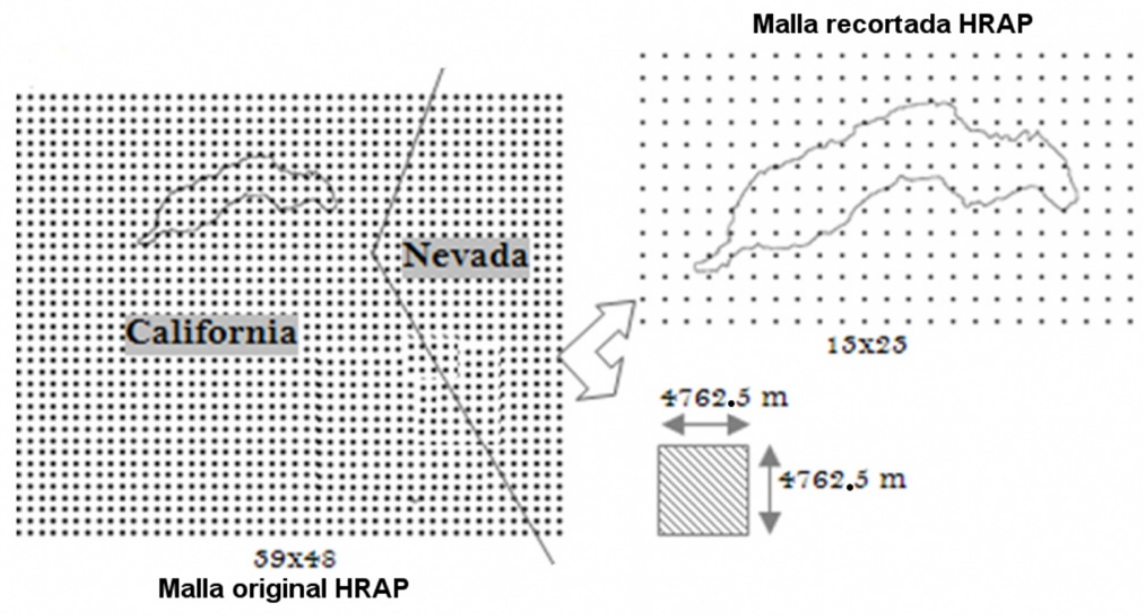

Figura 6. Malla de precipitaciones y temperaturas para un periodo de 1987 a 2002 recortadas al área de estudio. Fuente: Elaboración propia.

En la calibración del modelo hidrológico se ha usado información de caudales horarios instantáneos obtenidos de la estación de control NFDC1 (figura 1).

\section{Submodelo de fusión de nieve}

El modelo TETIS cuenta con un submodelo de fusión de nieve basado en el método grado-día (Hock, 2003). Según Vélez (2003), se eligió este método por ser ampliamente utilizado por su sencillez, por requerir poca información y por ser fácilmente adaptable a los modelos lluvia-escorrentía. El submodelo considera en su conceptualización una componente que toma en cuenta la acumulación de nieve en forma de altura equivalente de agua (SWE, por sus siglas en inglés) y otra para realizar la fusión de nieve empleando la siguiente ecuación: 


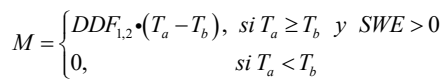

donde $\mathrm{M}$ es la tasa de fusión de nieve $(\mathrm{mm}), \mathrm{DDF}_{1,2}$ es el factor de fusión grado-día, $\mathrm{T}$ es la temperatura del aire $\left({ }^{\circ} \mathrm{C}\right)$ y $\mathrm{Tb}$ es la temperatura base a partir de la cual se define si hay fusión o acumulación de la nieve y varía entre $0{ }^{\circ} \mathrm{C}$ y $2{ }^{\circ} \mathrm{C}$ (DeWalle \& Rango, 2008). La SWE almacenada a nivel de celda es distribuida espacialmente usando el algoritmo de interpolación inverso de la distancia al cuadrado (IDW, por sus siglas en inglés). El submodelo de fusión de nieve se ha calibrado usando datos diarios de SWE medidas en las estaciones telemétricas de medición de nieve (SNOTEL, por sus siglas en inglés) (figura 1). La SWE representan la lámina de agua que se obtendría al fundirse la nieve acumulada.

\section{Metodología}

La metodología propuesta en este trabajo combina la calibración manual de los parámetros del submodelo de fusión de nieve y la calibración automática de los parámetros del modelo hidrológico. Esta metodología es llevada a cabo en tres fases que se muestran en la figura 7. En la fase I se ha seleccionado un evento con input solo por lluvia y se realizó una primera calibración manual con la finalidad de identificar los parámetros hidráulicos que proporcionan una alta sensibilidad al modelo. Es decir, se ha realizado un análisis de sensibilidad variando los parámetros dentro del rango conceptualmente permitido en la literatura científica.

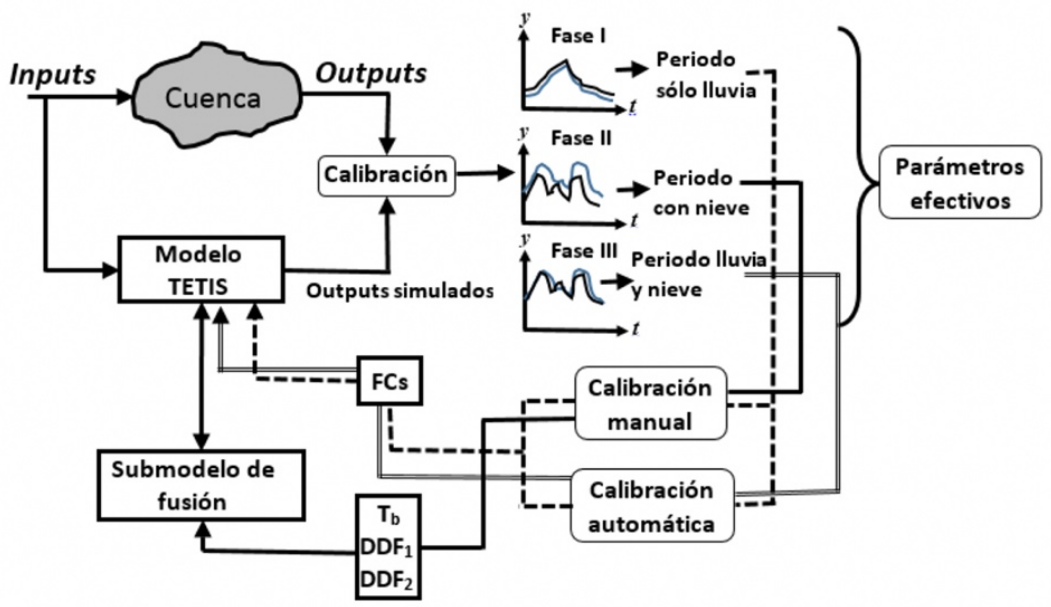

Figura 7. Metodología implementada en la calibración del modelo lluvia-escorrentía y el submodelo de fusión de nieve. Fuente: Elaboración propia.

En la fase II, se ha realizado la calibración manual de los parámetros del modelo de fusión de nieve fijando los parámetros efectivos obtenidos en la fase I. Por último, en la fase III se realizó una recalibración de los parámetros hidráulicos de la fase I fijando los parámetros del submodelo fusión de nieve obtenidos en la fase II.

El modelo TETIS calibra automáticamente nueve parámetros y cuatro variables de estado utilizando el algoritmo Shuffled Complex Evolution de la Universidad de Arizona (SCE-UA) propuesto por Duan et al. (1992) y Sorooshian et al. (1993). La calibración se realiza a través de nueve factores correctores (FC), que corrigen de forma global los parámetros de producción y traslación a nivel de celda (Francés et al., 2007) 
(tabla 1). En el caso del submodelo de fusión de nieve, la calibración de sus tres parámetros se realiza de forma directa al ser valores homogéneos en toda la subcuenca (tabla 1).

Tabla 1. Parámetros a calibrar del modelo hidrológico TETIS y el submodelo de fusión de nieve.

\begin{tabular}{|c|c|c|}
\hline Parámetro & Factor corrector & Parámetros efectivos \\
\hline \multicolumn{3}{|c|}{ Modelo hidrológico } \\
\hline Almacenamiento estático & $\mathrm{FC}_{1}$ & $H u_{(i)}{ }^{*}=\mathrm{FC}_{1} \cdot \mathrm{Hu}$ \\
\hline Índice de cobertura vegetal & $\mathrm{FC}_{2}$ & $\Lambda^{*}=\mathrm{FC}_{2} \cdot \lambda_{\mathrm{V}}$ \\
\hline Capacidad de infiltración & $\mathrm{FC}_{3}$ & $k_{s(i)^{*}}{ }=\mathrm{FC}_{3} \cdot \mathrm{k}_{s}$ \\
\hline Velocidad de escurrimiento superficial & $\mathrm{FC}_{4}$ & $\underline{\mathrm{u}}_{(\mathrm{i})}{ }^{*}=\mathrm{FC}_{4} \cdot \mathrm{u}$ \\
\hline Capacidad de percolación & $\mathrm{FC}_{5}$ & $k_{p(i)^{*}}=\mathrm{FC}_{5} \cdot \mathrm{k}_{\mathrm{p}}$ \\
\hline Velocidad de interflujo & $\mathrm{FC}_{6}$ & $k_{s s(i)}{ }^{*}=\mathrm{FC}_{6} \cdot \mathrm{k}_{s s}$ \\
\hline Capacidad de salida de flujo al acuífero & $\mathrm{FC}_{7}$ & $k_{p s(i)^{*}}=\mathrm{FC}_{7} \cdot \mathrm{k}_{\mathrm{sa}}$ \\
\hline Velocidad del flujo base & $\mathrm{FC}_{8}$ & $k_{s a(i)}{ }^{*}=\mathrm{FC}_{8} \cdot \mathrm{k}_{\mathrm{ps}}$ \\
\hline Velocidad en el cauce & $\mathrm{FC}_{9}$ & $\mathrm{~V}_{(\mathrm{t})} *=\mathrm{FC} \cdot \mathrm{V}_{(\mathrm{t})}$ \\
\hline \multicolumn{3}{|c|}{ Submodelo de fusión de nieve } \\
\hline Factor de fusión grado-día & $D D F_{1}$ & $D D F_{1}^{*}$ \\
\hline Factor de fusión grado-día con lluvia & $D D F_{2}$ & $D D F_{2}{ }^{*}$ \\
\hline Temperatura base & $T_{b}$ & $T_{b}{ }^{*}$ \\
\hline
\end{tabular}

Fuente. Elaboración propia.

La función objetivo seleccionada para el proceso de calibración ha sido el índice de eficiencia de Nash y Sutcliffe (NSE, por sus siglas en inglés), al ser el más usado en la calibración de modelos hidrológicos (Eckhardt \& Arnold, 2001; Eckhardt, Haverkamp, Fohrer \& Frede, 2002; Peterson \& Hamlett, 1998). El NSE se calcula a través de la ecuación siguiente:

$$
N S E=1-\sum_{t=1}^{T} \frac{\left(Q_{t, \text { sim }}-Q_{t, o b s}\right)^{2}}{\left(Q_{t, o s s}-\bar{Q}\right)^{2}}
$$

donde $Q_{t, s i m}$ es el caudal simulado, $Q_{t, o b s}$ es el caudal observado y $\bar{Q}$ es el caudal observado medio.

\section{Resultados y Discusión}

La calibración se ha realizado usando los datos de caudales horarios observados en la estación de control NFDC1, ubicada en la desembocadura de la subcuenca del río American (figura 1). Para la fase I se seleccionó un periodo en primavera comprendido del 20 de mayo de 1990 al 31 de julio de 1990 con una precipitación acumulada de $158.92 \mathrm{~mm}$. En esta fase se detectó que el modelo es muy sensible a los parámetros de capacidad de infiltración, capacidad de percolación y la velocidad de interflujo. El NSE obtenido en esta fase con el algoritmo SCE-UA ha sido de 0.92 (figura 8). 


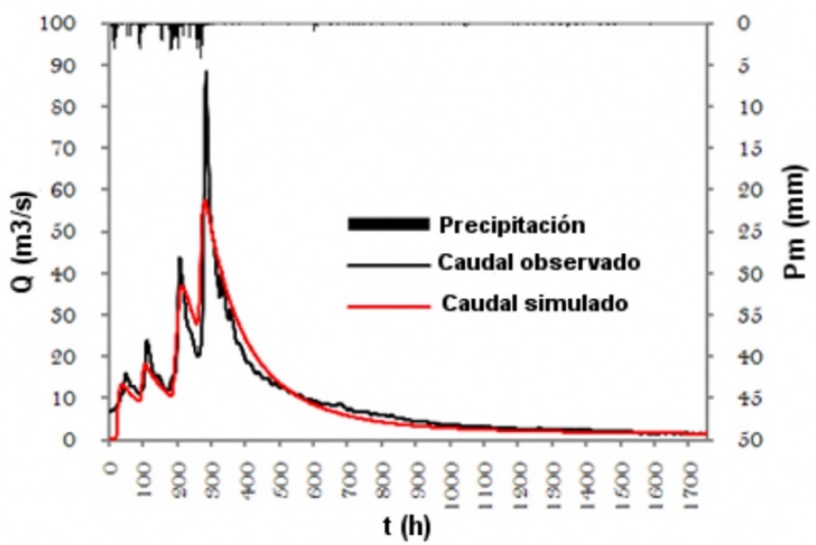

Figura 8. Resultado obtenido en la Fase I de la calibración de los parámetros de modelo hidrológico. Fuente: Elaboración propia.

La fase II se realizó de forma manual a prueba y error usando varias combinaciones de los parámetros $\mathrm{T}_{\mathrm{b}}, \mathrm{DDF}_{1}$ y $\mathrm{DDF}_{2}$. En este caso se seleccionaron tres periodos de calibración: con baja, media y alta presencia de nieve. Los parámetros que han obtenido las mejores eficiencias en la simulación de las SWE a nivel de celda en las dos estaciones SNOTEL son: $\mathrm{T}_{\mathrm{b}}=2.8, \mathrm{DDF}_{1}=2.7$ y $\mathrm{DDF}_{2}=5.0$. En la figura 9 se presentan los resultados obtenidos para el periodo de alta presencia de nieve. En general, los mejores resultados en la simulación de la fusión y acumulación de la nieve se han obtenido en las zonas más elevadas y para los episodios con media y alta presencia de nieve. Estos resultados coinciden con los obtenidos por Shamir \& Georgakakos (2006) para la misma área de estudio.
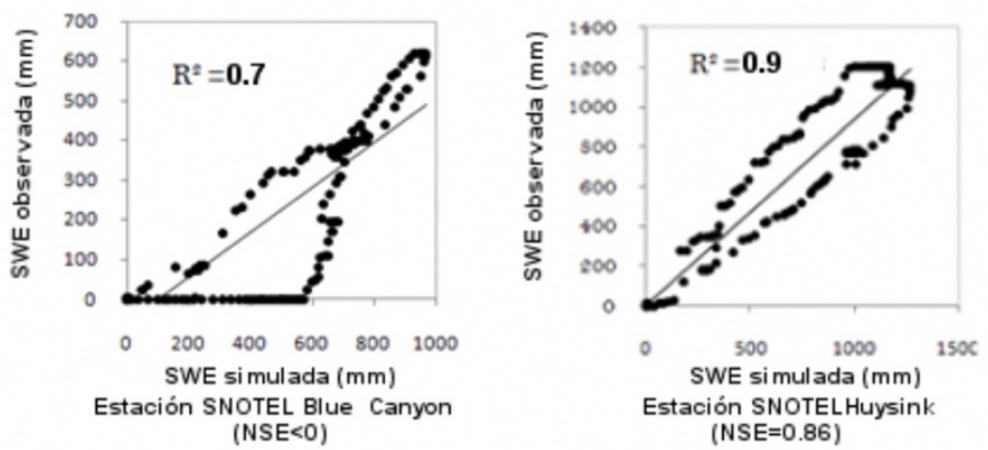

Figura 9. Resultados de la calibración manual del modelo de fusión de nieve en la subcuenca del río American (Periodo: 1ํo de noviembre de 1992-31 de julio de 1993).

Fuente: Elaboración propia.

La fase III consistió en recalibrar de manera automática los parámetros del modelo hidrológico, fijando los parámetros del submodelo de fusión de nieve. Para ello, se seleccionaron tres periodos con la finalidad de obtener parámetros efectivos más robustos (tabla 2). En las figura 10, 11 y 12 se presentan los resultados obtenidos por calibración automática para cada uno de los periodos seleccionados. 
Tabla 1. Episodios seleccionados para el calentamiento y la calibración del modelo en la Fase III.

\begin{tabular}{cll}
\hline Periodo & Calentamiento & Calibración \\
\hline I & $01 /$ dic/1988-01/feb/1989 & $01 /$ feb/1989-30/jun/1989 \\
II & $01 /$ nov/1990-01/feb/1991 & $01 /$ feb/1991-31/jul/1991 \\
III & $01 /$ ago/1992-01/nov/1992 & $01 /$ nov/1992-31/jul/1993 \\
\hline
\end{tabular}

Fuente. Elaboración propia.

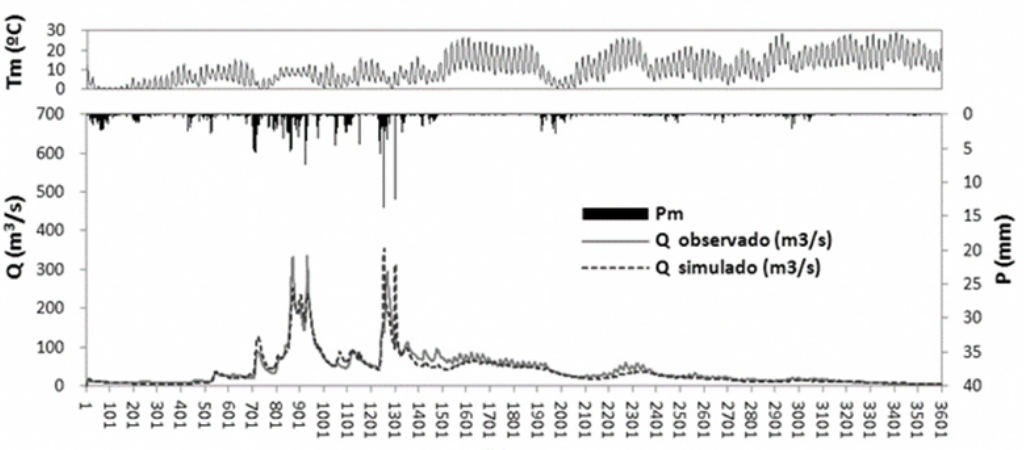

$t(h)$

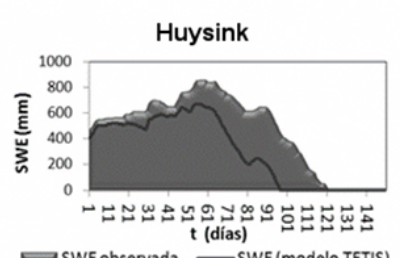

WSWEobservada - SWE (modelo TETIS)

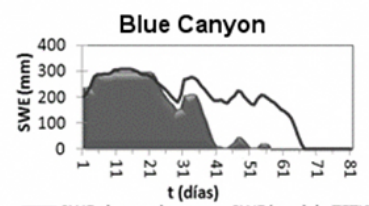

Figura 10. Resultados obtenidos para el período I en la simulación de los caudales (NSE = 0.88) y la acumulación de nieve (NSE-Huysink $=0.4$ y NSE-Blue Canyon $=0.5$ ).

Fuente: Elaboración propia.
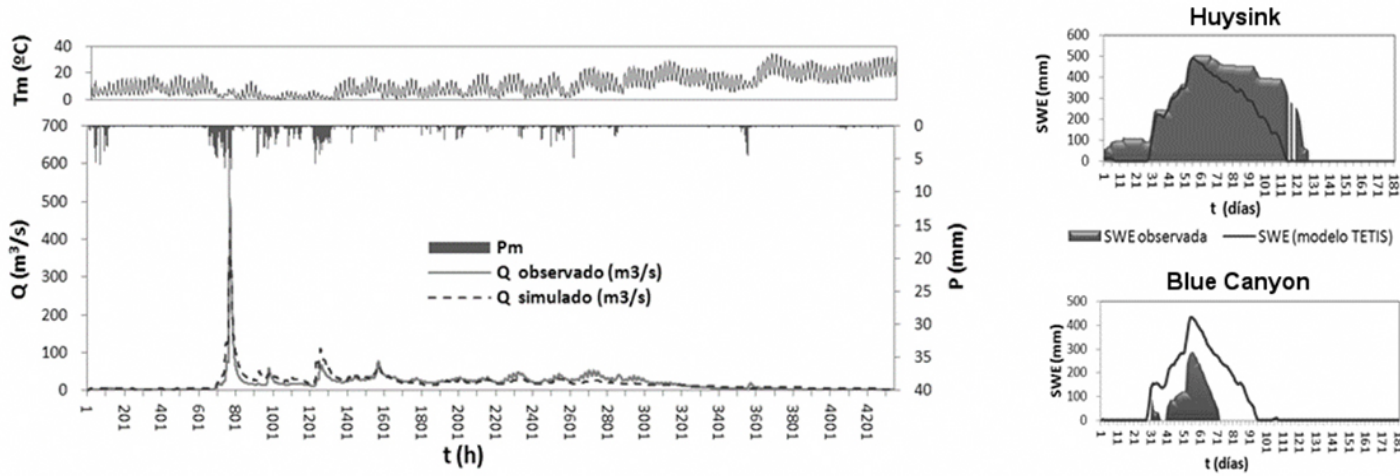

—SWE observada - SWE(modelo TETIS)

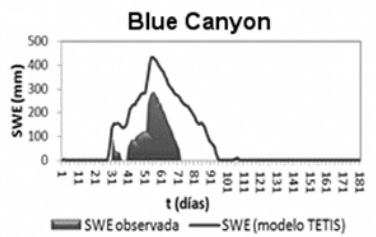

Figura 11. Resultados obtenidos para el período II en la simulación de los caudales (NSE $=0.84$ ) y la acumulación de nieve (NSE-Huysink = 0.6 y NSE-Blue Canyon $<0$ ).

Fuente: Elaboración propia. 


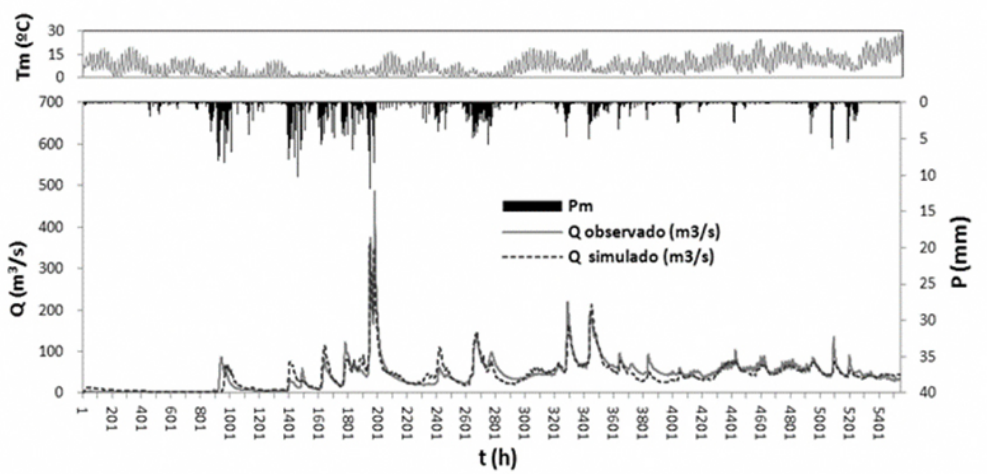

$\mathrm{t}$ (h)

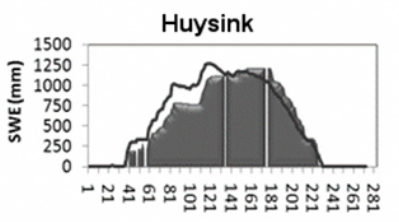

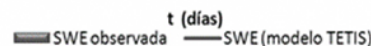

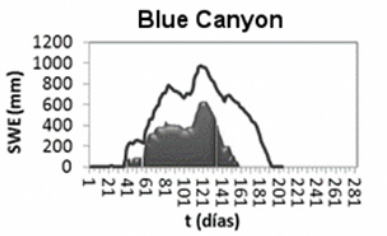

Figura 12. Resultados obtenidos para el período III en la simulación de los caudales (NSE $=0.85)$ y la acumulación de nieve (NSE-Huysink $=0.8$ y NSE-Blue Canyon < 0 )

Fuente: Elaboración propia.

En los tres periodos las eficiencias obtenidas son muy buenas, es por ello que, como criterio para seleccionar el conjunto de parámetros efectivos más robustos, se decidió realizar una simulación continúa usando el periodo comprendido entre el 1ㅇ de febrero de 1989 y 31 de julio de 1993. Los NSE obtenidos en esta simulación se muestran en la tabla 3. Con base en estos resultados, los parámetros seleccionados corresponden a los obtenidos para el periodo II. En la tabla 4 se presenta la variabilidad espacial de los parámetros efectivos obtenida al realizar la tres fases de la calibración, según la metodología propuesta.

Tabla 3. Conjunto de FC y NSE obtenidos en la simulación continua realizada para seleccionar los parámetros efectivos.

\begin{tabular}{ccccccccccc}
\hline & FC1 & FC2 & FC3 & FC4 & FC5 & FC6 & FC7 & FC8 & FC9 & NSE \\
\hline I & 1.40 & 1.21 & 0.99 & 8.00 & 0.58 & 260.98 & 0.00 & 38.17 & 0.50 & 0.74 \\
II & 1.47 & 1.13 & 0.74 & 8.00 & 0.90 & 290.81 & 0.00 & 29.85 & 0.60 & 0.77 \\
II & 1.30 & 1.06 & 0.93 & 5.00 & 0.78 & 305.11 & 0.00 & 57.17 & 0.50 & 0.74 \\
\hline
\end{tabular}

Fuente. Elaboración propia.

Tabla 4. Muestra los rangos de variación espacial de los parámetros efectivos conseguidos en el proceso de la calibración.

\begin{tabular}{lr}
\hline Parámetro & \multicolumn{2}{c}{$\begin{array}{c}\text { Rango de parámetro } \\
\text { efectivos }\end{array}$} \\
\hline \multicolumn{1}{c}{ Modelo hidrológico } \\
\hline Almacenamiento estático (mm) & $112.43-200$ \\
Índice de cobertura vegetal (adimensional) & $0.5-1.38$ \\
Capacidad de infiltración (mm/h) & $17.7-30.02$ \\
Velocidad de escurrimiento superficial (m/s) & $0.69-1.84$ \\
Capacidad de percolación (mm/h) & $4.80-14.47$ \\
Velocidad de interflujo (mm/h) & $5.8 \times 10^{-3}-12.3 \times 10^{-3}$ \\
Capacidad de salida de flujo al acuífero (mm/h) & $0.0-1.96$ \\
Velocidad del flujo base (mm/h) & $32.3-58.01$ \\
Velocidad en el cauce (m/s) & $0.87-1.99$ \\
\hline \multicolumn{2}{c}{ Submodelo de fusión de nieve }
\end{tabular}




\begin{tabular}{lc}
\hline Factor de fusión grado-día $\left(\mathrm{mm} /{ }^{\circ} \mathrm{Cd}\right)$ & $3.39-3.51$ \\
Factor de fusión grado-día con lluvia $\left(\mathrm{mm} /{ }^{\circ} \mathrm{Cd}\right)$ & $3.17-4.52$ \\
Temperatura base $\left({ }^{\circ} \mathrm{C}\right)$ & $2.05-2.54$ \\
\hline
\end{tabular}

Fuente. Elaboración propia.

Una limitación de los modelos hidrológicos es su extrapolaridad, es decir, no hay garantía de que el modelo tenga un buen rendimiento cuando se usa en condiciones diferentes de las presentes en la calibración (Gan \& Burges, 1990; Vélez, Puricelli, López \& Francés, 2009). Por consiguiente, el proceso de validación de un modelo tiene la función de demostrar que los componentes hidrológicos dominantes en una cuenca han sido adecuadamente determinados y que el rendimiento de predicción deseada puede ser alcanzado y fiable (Andersen, Refsgaard \& Jensen, 2001; Klemes, 1988; Refsgaars \& Knudsen, 1996; Senarath, Ogden, Downer \& Sharif, 2000). Para lo anterior se realizó validación temporal utilizando el periodo comprendido del 1ㅇ de octubre de 1994 al 30 de septiembre de 1997. El NSE obtenido en la simulación de los caudales observados ha sido de 0.81 y NSE superiores a 0.7 en la acumulación de nieve (Huysink: NSE $=0.83$ y Blue Canyon: NSE $=0.73$ ) (figura 13).

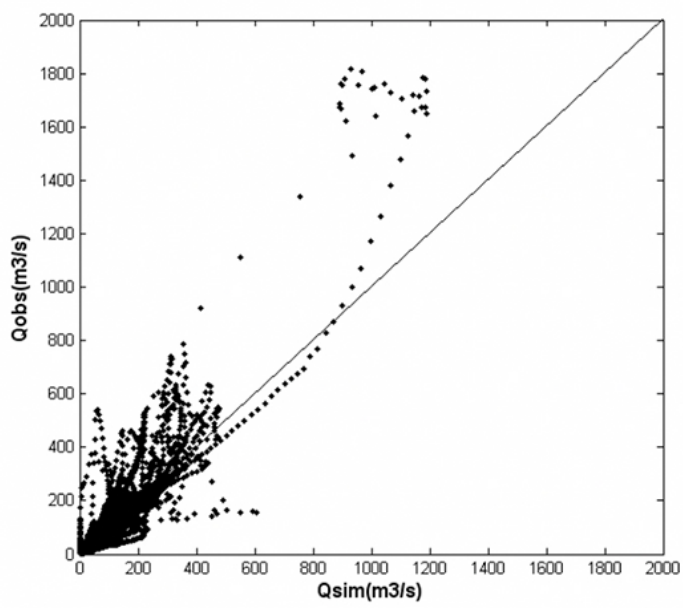

a)

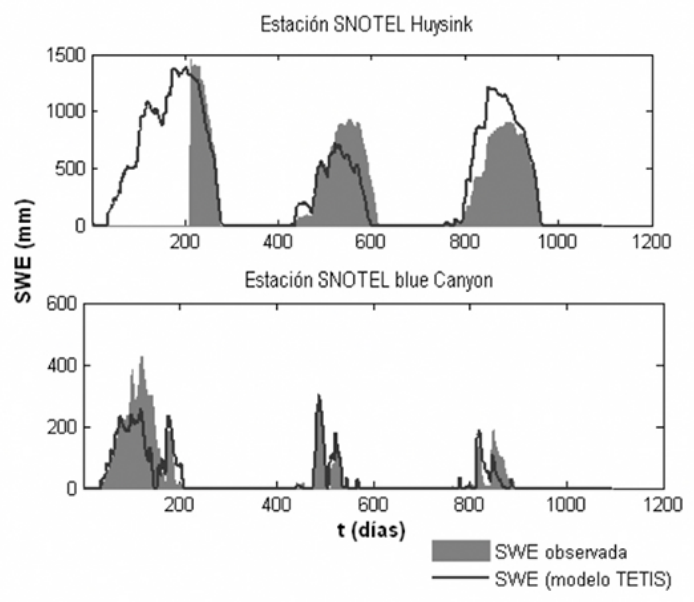

b)

Figura 13. Resultados obtenidos en la validación temporal del modelo utilizando los FC calibrados con la metodología implementada (a:
caudales y b: acumulación de nieve).
Fuente: Elaboración propia.

En este proceso de validación se realizó también la comparación de la distribución espacial de la acumulación de nieve que realiza el modelo contra 202 imágenes de satélite de cubiertas de nieve proporcionadas por la NOAA/NWS. Se observó en términos generales una buena aproximación del submodelo con sobreestimaciones inferiores al $27 \%$ y subestimaciones inferiores al $6 \%$ para los casos analizados.

\section{Conclusiones}

La combinación de la calibración manual y automática implementada en tres fases como una alternativa para facilitar el proceso de calibración de un modelo lluvia-escorrentía ha permitido obtener un conjunto de parámetros efectivos capaz de simular con buenos resultados los caudales observados en la desembocadura y la acumulación de nieve en una cuenca de alta montaña. Además, se ha podido observar que existen múltiples combinaciones de parámetros que proporcionan una adecuada representación del 
sistema, dando indicios de que la tendencia en la calibración de los modelos determinísticos deberá ser multiobjetivo para seleccionar la mejor opción y evitar enmascarar los resultados de priorizar un único elemento del sistema. En el caso de los resultados obtenidos en el proceso de validación mostraron una buena capacidad del modelo para reproducir el comportamiento del sistema real en condiciones diferentes a las presentes en los periodos usados en la calibración.

\section{Agradecimientos}

Esta investigación ha sido apoyada por la Dirección General de Educación Superior Universitaria (DGESU) de la Secretaría de Educación Pública, a través de su Programa para el Desarrollo Profesional Docente (Folio prodep: UGTO-PTC 613) y por la División de Ingenierías de la Universidad de Guanajuato.

\section{Referencias}

Andersen, J., Refsgaard, J. C., \& Jensen, K. H. (2001). Distributed hydrological modelling of the Senegal River Basin model construction and validation. Journal of Hydrology, 247(3), 200-214. doi: https://doi.org/10.1016/S0022-1694(01)00384-5

Chapman, T. (1970). Optimization of a rainfall-runoff model for an arid zone catchment. United Nations Educational, Scientific and Cultural Organization (UNESCO) Publications, 96, 126-144.

Duan, Q., Sorooshian, S., \& Gupta, V. (1992). Effective and Efficient Global Optimization for Conceptual RainfallRunoff Models. Water Resources Research, 28(4), 1015-1031.

Dawdy, D. R., \& O'Donnell, T. (1965). Mathematical models of catchment behavior. Journal of the Hydraulics Division, 91(4), 113-137.

DeWalle, D. R., \& Rango, A. (2008). Principles of snow hydrology. Cambridge: Cambridge University Press. doi: https://doi.org/10.1017/CB09780511535673

Eckhardt, K., \& Arnold, J. G. (2001). Automatic calibration of a distributed catchment model. Journal of Hydrology, 251(1), 103-109. doi: https://doi.org/10.1016/S0022-1694(01)00429-2

Eckhardt, K., Haverkamp, S., Fohrer, N., \& Frede, H. G. (2002). SWAT-G, a version of SWAT99.2 modified for application to low mountain range catchments. Physics and Chemistry of the Earth, Parts $A / B / C, 27(9), 641$ 644. doi: https://doi.org/10.1016/S1474-7065(02)00048-7

Francés, F., Vélez, J. I., \& Vélez, J. J. (2007). Split-parameter structure for the automatic calibration of distributed hydrological models. Journal of Hydrology, 332(1), 226-240. doi: https://doi.org/10.1016/j.jhydrol.2006.06.032

Francés, F., Vélez, J. I., Vélez, J. J., Puricelli, M., Montoya, J. J., Múnera, J. C., Bussi, G., Medici, C., \& Orozco, I. (2014). Description of the distributed conceptual hydrological model tetis v.8. España: Universitat Politècnica de València.

Gan, T. Y., \& Burges, S. J. (1990). An assessment of a conceptual rainfall-runoff model's ability to represent the dynamics of small hypothetical catchments: 1 . models, model properties, and experimental design. Water Resources Research, 26(7), 1595-1604. doi: https://doi.org/10.1029/WR026i007p01595

Gray, D. M. (1970). Handbook on the principles of hydrology; with special emphasis directed to Canadian conditions in the discussions, applications, and presentation of data. Ottawa, Canada: Water Information Center, inc.

Gupta, V. K., \& Sorooshian, S. (1985). The automatic calibration of conceptual catchment models using derivativebased optimization algorithms. Water Resources Research, 21(4), 473-485. doi: https://doi.org/10.1029/WR021i004p00473

Hock, R. (2003). Temperature index melt modelling in mountain areas. Journal of Hydrology, 282(1-4), 104-115. doi: https://doi.org/10.1016/S0022-1694(03)00257-9

Hendrickson, J. D., Sorooshian, S., \& Brazil, L. E. (1988). Comparison of Newton-type and direct search algorithms for calibration of conceptual rainfall-runoff models. Water Resources Research, 24(5), 691-700. doi: https://doi.org/10.1029/WR024i005p00691 
Jansson, P., Hock, R., \& Schneider, T. (2003). The concept of glacier storage: a review. Journal of Hydrology, 282(14), 116-129. doi: https://doi.org/10.1016/S0022-1694(03)00258-0

Jeton, A. E., Dettinger, M. D., \& LaRue Smith, J. (1996). Potential effects of climate change on streamflow, Eastern and Western slopes of the Sierra Nevada, California and Nevada. Sacramento, California: US Department of the interior.

Johnston, P. R., \& Pilgrim, D. H. (1976). Parameter optimization for watershed models. Water Resources Research, 12(3), 477-486. doi: https://doi.org/10.1029/WR012i003p00477

Klemes, V. (1988). A hydrological perspective. Journal of Hydrology, 100(1-3), 3-28. doi: https://doi.org/10.1016/0022-1694(88)90179-5

Madsen, H. (2000). Automatic calibration of a conceptual rainfall-runoff model using multiple objectives. Journal of Hydrology, 235(3-4), 276-288. doi: https://doi.org/10.1016/S0022-1694(00)00279-1

Monro, J. (1971). Direct search optimization in mathematical modeling and a watershed model application. Technical Memorandum, NWS Hydro-12, National. Oceanic and Atmos. Admin., U.S. Dep. of Commerc, Silver Spring, 1, 1-52.

Moreda, F., Cong, S., Schaake, J., \& Smith, M. (2006). Gridded rainfall estimation for distributed modeling in western mountainous areas. Advancing earth and space science (AGU) Spring Meeting, 23, 32.

Nash, J. E., \& Sutcliff, J. V. (1970). River flow forecasting through conceptual models part I a discussion of principles. Journal of Hydrology, 10(3), 282-290. doi: https://doi.org/10.1016/0022-1694(70)90255-6

Pandey, G. R., Cayan, D. R., \& Georgakakos, K. P. (1999). Precipitation structure in the Sierra Nevada of California during winter. Journal Geophysical Research Atmospheres, 104(D-10), 2019-12030. doi: https://doi.org/10.1029/1999JD900103

Peterson, J. R., \& Hamlett, J. M. (1998). Hydrologic Calibration of The Swat Model In A Watershed Containing Fragipan Soils. Journal of the American Water Resources Association (JAWRA), 34(3), 531-544. doi: https://doi.org/10.1111/j.1752-1688.1998.tb00952.x

Pickup, G. (1977). Testing of efficiencies of algorithms and strategies for automatic calibration of rainfall-runoff models. Hydrological Sciences Bulletin, 22(2), 257-274. doi: https://doi.org/10.1080/02626667709491716

Refsgaars, J. C., \& Knudsen, J. (1996). Operational validation and intercomparison of different types of hydrological models. Water Resources Research, Surface Water and Climate, 32(7), 2189-2202. doi: https://doi.org/10.1029/96WR00896

Senarath, S. U. S, Ogden, F. L., Downer, C. W., \& Sharif, H. O. (2000). On the calibration and verification of twodimensional distributed, Hortonian, continuous watershed models. Water Resources Research, Surface Water and Climate, 36(6), 1495-1510. doi: https://doi.org/10.1029/2000WR900039

Shamir, E., \& Georgakakos, K. P. (2007). Estimating snow depletion curves for American River basins using distributed snow modeling. Journal of Hydrology, 334(1-2), 162-173. doi: https://doi.org/10.1016/j.jhydrol.2006.10.007

Shamir, E., \& Georgakakos, K. P. (2006). Distributed snow accumulation and ablation modeling in the American River basin. Advances in Water Resources, 29(4), 558-570. doi: https://doi.org/10.1016/j.advwatres.2005.06.010

Smith, M., Koren, V., Zhang, Z., Moreda, F., Cui, Z., Cosgrove, B., Mizukami, N., Kitzmiller, D., Ding, F., Reed, S., Anderson, E., Schaake, J., Zhang, Y., Andréassian, V., Perrin, C., Coron, L., Valéry, A., Khakbaz, B., Sorooshian, S., Behrangi, A., Imam, B., Hsu, K. L., Todini, E., Coccia, G., Mazzetti, C., Ortiz Andres, E., Francés, F., Orozco, I., Hartman, R., Henkel, A., Fickenscher, P., \& Staggs, S. (2013). The distributed model intercomparison project phase 2: Experiment design and summary results of the western basin experiments. Journal of Hydrology, 507, 300-329. doi: https://doi.org/10.1016/j.jhydrol.2013.08.040

Sorooshian, S., Duan, Q., \& Gupta, V. K. (1993). Calibration of rainfall-runoff models: application of global optimization to the Sacramento soil moisture accounting model. Water Resources Research, 29(4), 11851194. doi: https://doi.org/10.1029/92WR02617

Sorooshian, S. (1981). Parameter estimation of rainfall-runoff models with heteroscedastic streamflow errors-The noninformative data case. Journal of Hydrology. 52(1-2), 127-138. doi: https://doi.org/10.1016/00221694(81)90099-8 
Vélez Upegui, J. J. (2003). Desarrollo de un modelo distribuido de predicción en tiempo real para eventos de crecidas (Tesis de Doctorado). Universidad Politécnica de Valencia, España.

Vélez, J. J., Puricelli, M., López Unzu, F., \& Francés, F. (2009). Parameter extrapolation to ungauged basins with a hydrological distributed model in a regional framework. Hydrology and Earth System Sciences, 13, 229246. doi: https://doi.org/10.5194/hess-13-229-2009

Yapo, P. O., Gupta, H. V., \& Sorooshian, S. (1998). Multi-objective global optimization for hydrologic models. Journal of Hydrology, 204(1-4), 83-97. doi: https://doi.org/10.1016/S0022-1694(97)00107-8 